\title{
Modelled current distribution in cervical squamous tissue
}

\author{
D C Walker, B H Brown, R H Smallwood, D R Hose and D M Jones \\ Department of Medical Physics and Clinical Engineering, University of Sheffield, Floor I, \\ Royal Hallamshire Hospital, Glossop Road, Sheffield, S10 2JF, UK \\ E-mail: mpp98dcw@sheffield.ac.uk
}

Received 25 May 2001, in final form 26 October 2001

Published 28 January 2002

Online at stacks.iop.org/PM/23/159

\begin{abstract}
The electrical properties of cervical squamous epithelium have been modelled in the frequency range $100 \mathrm{~Hz}$ to $10 \mathrm{MHz}$. The hierarchical modelling process comprises a cellular level stage, which includes detailed models of cells typical of different depths within the epithelium and a tissue model, which utilizes electrical properties obtained from the cellular models. The fit between the modelled and measured impedance spectra and the distribution of current with depth depends on the macroscopic model structure. Both the properties of the basement membrane and the presence of a surface mucus layer are shown to have a significant effect. The best fit with measured data is obtained when a $10 \mu \mathrm{m}$ thick, high-conductivity surface layer is included in the tissue model.
\end{abstract}

Keywords: electrical impedance tomography (EIS), modelling, epithelium, uterine cervix

\section{Introduction}

Clinical trials of an electrical impedance method of diagnosis for cervical neoplasia have been undertaken in Sheffield (Brown et al 2000). A tetrapolar technique was employed, whereby current was driven between two adjacent electrodes mounted on the tip of a pencil probe and the potential recorded between the second pair. The operating range of this system is $4.8-614 \mathrm{kHz}$. Good separation between normal and precancerous tissue has been obtained in terms of the low-frequency impedance, $R$ and the high-frequency impedance, $S$. There is also a distinct difference in the characteristic frequencies, $f_{c}$, or the point of maximum slope when the real part of the impedance is plotted against frequency for the two tissue types.

These changes in impedance are related to both cellular and structural changes within the tissue. Figure 1 shows a schematic interpretation of the progression from normal cervical epithelium, though the precancerous stages known as cervical intraepithelial neoplasia (CIN) 


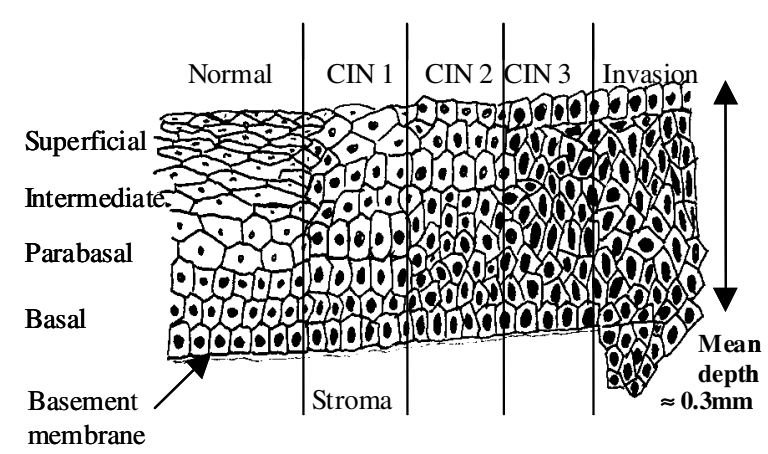

Figure 1. Changes in tissue structure associated with the progression of CIN in cervical squamous epithelium.

to invasion. The changes include an increased nuclear-cytoplasmic ratio, loss of the layer of flattened cells close to the surface and an increase in the volume of extra-cellular space (White and Gohari 1984, Sorenson et al 1991). The frequency-dependent electrical behaviour of a single cell is determined by the electrical properties of the membrane, intra- and extra-celluar spaces and nucleus (Schwan 1957). The complex electrical impedance of tissue will also be determined by the dimensions, internal structure and arrangements of the constituent cells associated with different tissue pathologies.

Introduction of routine cervical screening in the form of the Pap smear test has significantly improved the early detection rate and thus the opportunity for successful treatment. However, this test remains highly subjective and consequently has low sensitivity. The development of a more accurate and faster method of diagnosis is therefore desirable. The purpose of the modelling outlined in this paper is to gain further understanding of the pattern of current flow through cervical epithelium and the underlying tissue, and hence aid in improvements to the current data collection system. We have already developed a cervical tissue model, which can reproduce the pattern in change in impedance spectra for normal and CIN 3 tissue observed in vivo (Walker et al 2000). This paper examines in more detail the sensitivity of this macroscopic tissue model to the choice of input parameters and discusses the resulting change in the distribution of current flow with depth.

\section{Methods}

The finite element technique is a numerical analysis method routinely used in the solution of physics field problems. In the case of electrical problems, it is used to calculate an approximate solution for the potential distribution within a volume partitioned into elements. In summary, finite element analysis attempts to solve the equation:

$$
\left\lfloor K_{g}\right\rfloor\{\phi\}=\{I\}
$$

where $\phi$ is the vector of nodal voltages, $I$ is the vector of applied currents and $K_{g}$ is a matrix of internal complex conductivities (known as the global stiffness matrix). The theory of this technique and examples of applications to electrical biomedical problems can be found in Miller and Henriquez (1990). If the electrical properties of the various materials in our cell or tissue model are known (or assumed), for any given set of applied currents, the resulting potential at any point on the model boundary, and hence the transfer impedance, can be calculated. 


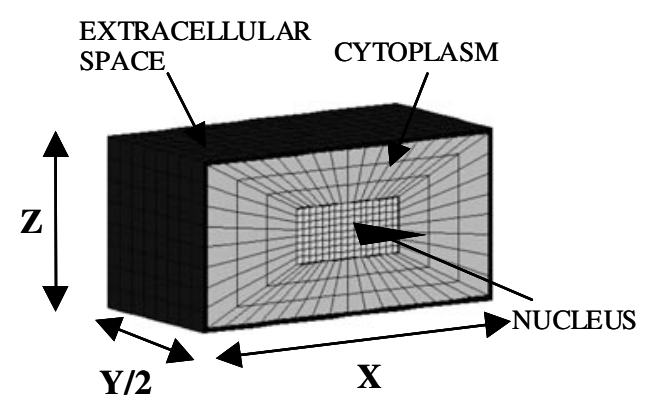

Figure 2. Cross section of finite element model of single cell.

Table 1. Dimensions of cellular compartments used in model construction $(\mathrm{N}: \mathrm{C}=$ nuclearcytoplasmic ratio, ECS = extracellular space).

\begin{tabular}{|c|c|c|c|c|c|}
\hline \multirow[b]{2}{*}{ Layer no } & \multicolumn{3}{|c|}{ Cell dimensions $(\mu \mathrm{m})$} & \multirow[b]{2}{*}{$\mathrm{N}: \mathrm{C}$ ratio } & \multirow[b]{2}{*}{ ECS width $(\mu \mathrm{m})$} \\
\hline & $X$ & $Y$ & $Z$ & & \\
\hline 1. Superficial & 65 & 65 & 15 & 0.01 & 0.05 \\
\hline 2. Intermediate & 50 & 50 & 15 & 0.01 & 0.1 \\
\hline 3. Parabasal & 30 & 30 & 15 & 0.05 & 0.2 \\
\hline 4. Basal & 15 & 15 & 15 & 0.2 & 0.4 \\
\hline
\end{tabular}

Table 2. Electrical properties used to model cellular compartments, derived from average values published in literature (Geddes and Baker 1967, Irimijiri et al 1978, Bordi et al 1993, Asami and Takashima 1989, Huang et al 1995).

\begin{tabular}{llc}
\hline Cellular compartment & Conductivity, $\sigma\left(\mathrm{S} \mathrm{m}^{-1}\right)$ & Relative Permittivity, $\varepsilon_{\mathrm{r}}$ \\
\hline Extracellular space & 1.1 & 72 \\
Cytoplasm & 0.6 & 86 \\
Nuclear interior & 0.8 & 145 \\
\hline
\end{tabular}

Due to computing resource limitations, it is not possible to model the dimensions of tissue 'seen' by our impedance measurement system with cellular level detail. Our approach to this problem was to use a hierarchical method of modelling. Finite element models of cells with dimensions typical of four different depths in normal cervical epithelium were constructed, as shown in figure 2. The dimensions of these compartments were chosen to mimic the true cell structures, based on published studies of the ultrastructure of cervical epithelium (Friedrich 1973), and observation of histological tissue sections. Dimensions of cellular compartements used in constructing the cellular level models are given in table 1.

Electrical properties assigned to each cellular compartment (i.e., nucleus, nuclear and cytoplasmic membranes, intra- and extracellular space) were based on averages of values published in the literature, and are given in tables 2 and 3. Single cells were meshed together to form the 'bricked' pattern observed in normal epithelium. Currents in the frequency range $100 \mathrm{~Hz}-10 \mathrm{MHz}$ were applied at the boundaries of the model in the $x$ and $z$ planes (i.e. parallel and perpendicular to the long axis of the cell, which is usually aligned parallel to the epithelial surface). The resulting potentials on the appropriate boundary planes, and hence the electrical transfer conductivities in each direction were obtained, as shown in figure 3. The models are 


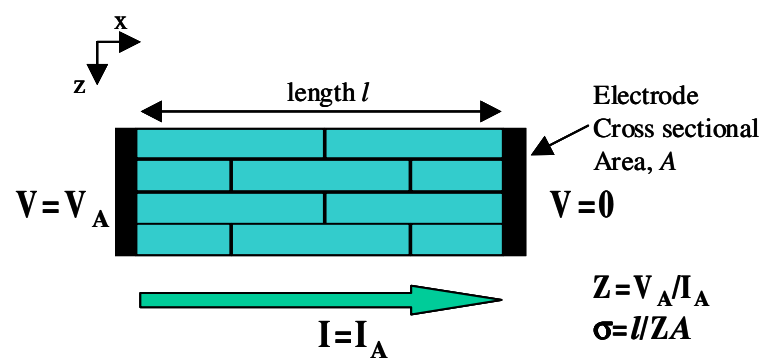

Figure 3. Method of obtaining $x$-direction electrical transfer properties for single cell type.

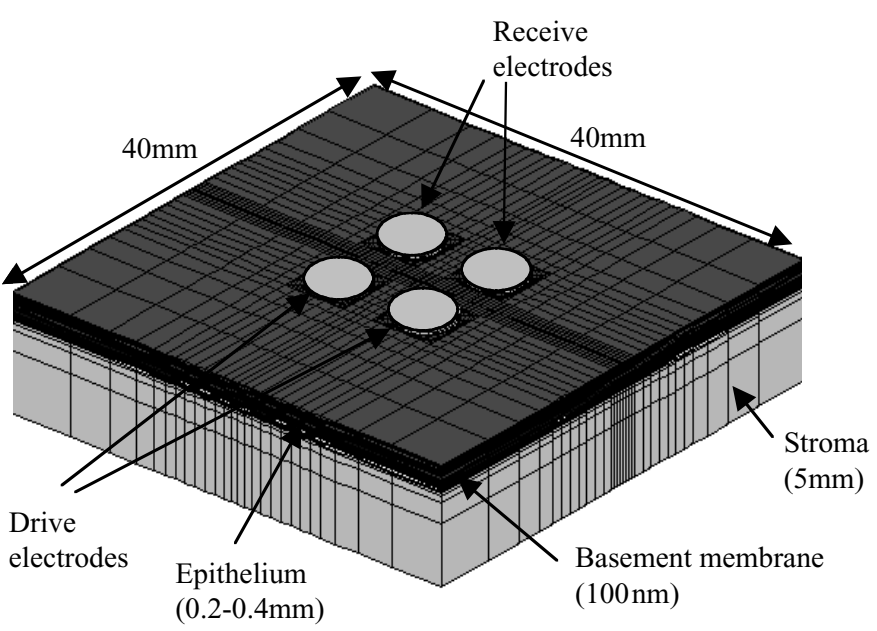

Figure 4. Macroscopic tissue model with surface electrode array.

Table 3. Electrical properties used to model membranes, derived from average values published in literature (Pethig and Kell 1987, Cole 1972, Kotnik and Miklavcic 2000. Irimijiri et al 1978, Bordi et al 1993).

\begin{tabular}{lcll}
\hline Membrane & Thickness $(\mathrm{nm})$ & Capacitance $\left(\mu \mathrm{F} \mathrm{m}^{-1}\right)$ & Conductivity $\left(\mu \mathrm{S} \mathrm{m}^{-1}\right)$ \\
\hline Cytoplasmic & 8.0 & 0.01 & 0.1 \\
Nuclear & 40.0 & 0.007 & 2000 \\
\hline
\end{tabular}

identical in all respects in the $x$ and $y$ planes, and hence the properties in these two directions will also be identical.

These properties were then assigned as electrical properties to epithelial layers in our macroscopic tissue model (Walker et al 2000). This model consisted of four epithelial layers representing superficial, intermediate, parabasal and basal cell types, underlying layers representing the basement membrane and stroma, and a surface layer of variable thickness, representing a thin layer of cervical mucus. The macroscopic model mesh is shown in figure 4.

The basement membrane consists of a $100 \mathrm{~nm}$ thick network of collagen type IV fibres, which provides support for the epithelium and attaches it firmly to the fibromuscular stroma. There is no published data for the electrical properties of these tissue layers, so models were 
Table 4. Properties assigned to basement membrane layer.

\begin{tabular}{lll}
\hline & Conductivity & Relative permittivity \\
\hline Set 1 & Frequency-dependent tendon & Frequency-dependent tendon \\
& properties, $0.3-0.4 \mathrm{~S} \mathrm{~m}^{-1}$ (Gabriel et al 1996b) & properties $10^{2}-10^{7}$ (Gabriel et al 1996b) \\
Set 2 & $1 \mu \mathrm{S} \mathrm{m}^{-1}$ & 90 \\
\hline
\end{tabular}

solved initially using the published conductivity and permittivity values for tendon (Gabriel et al 1996b) to represent basement membrane. In order to assess model sensitivity to the properties of this layer, solutions were also obtained using basement membrane conductivities of a similar order of magnitude to the very small values associated with cell membrane properties $\left(10^{-6} \mathrm{~S} \mathrm{~m}^{-1}\right)$ and a permittivity value of 90 , typical of many biological materials. This information is summarized in table 4. The conductivity of stroma was measured by placing an impedance probe directly onto freshly excised, unfixed healthy stroma obtained, with ethical committee approval and consent, from hysterectomy patients. This system measures only the real part of the impedance, so permittivity values used for stroma were those published in the literature for uterus (Gabriel et al 1996a). The mucus layer was assigned similar properties to those used for extracellular fluid.

Current was applied to drive electrodes in the macroscopic model in the frequency range $100 \mathrm{~Hz}-10 \mathrm{MHz}$ and voltages calculated at the receive electrodes in an identical arrangement to the in vivo tissue measurements. It is therefore possible to directly compare plots of transfer impedivity against frequency (i.e. the impedance spectra) obtained from the model with those obtained in vivo. The current flow through every node located on the zero potential boundary midway between the two drive electrodes was calculated using equation (2.1) and then integrated to give the total current flowing through each layer.

Various model parameters can be altered at either the cellular, or the macroscopic stage of the modelling process in order to assess the effect on the impedance spectrum or the current distribution. In this case, we have varied the macroscopic level parameters of epithelial thickness and depth of surface mucus layer. The distribution of thickesses of squamous epithelium in normal patients was obtained from the same histology sections used to obtain cellular morphological parameters. Thickesses were found to vary between 170 and $490 \mu \mathrm{m}$, with a mean value of $290 \mu \mathrm{m}$. Models were solved with total epithelial thicknesses of 200 , 300 and $400 \mu \mathrm{m}$. Arbitrary mucus layer thicknesses of 0 (no mucus layer), 10, 50 and $100 \mu \mathrm{m}$ were solved.

\section{Results}

\subsection{Impedance spectra}

The real part of the transfer impedivity characteristics of the four cell types in the $x-y$ plane (i.e. parallel to the surface of the epithelium) are shown in figure 5. The model cell dimensions used to obtain these properties are those given in table 1 and the electrical properties in tables 2 and 3. Figure 6 shows the impedance spectra obtained from the macroscopic model using these epithelial layer properties. The model was solved for two sets of basement membrane properties including a total epithelial depth of 200, 300 and $400 \mu \mathrm{m}$. In this case, a surface mucus layer was not included in the model. This figure also includes the mean and 95\% region calculated from 86 impedance spectra obtained from in vivo measurements on 23 patients whose tissue was diagnosed as normal. Diagnosis of the pathology of the tissue 


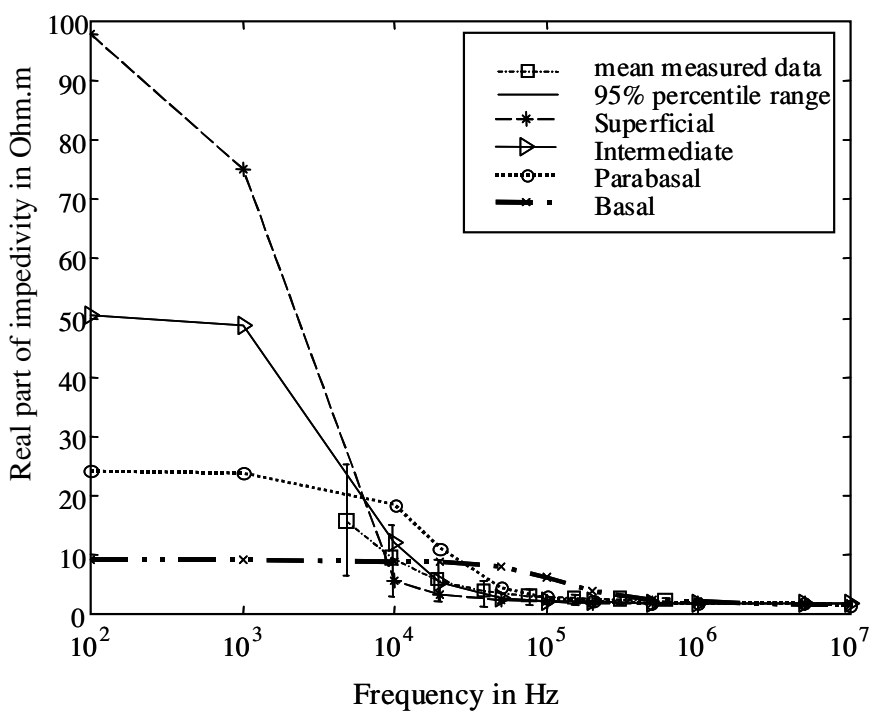

Figure 5. Real component of $x$-direction transfer impedance properties for different epithelial cell types.

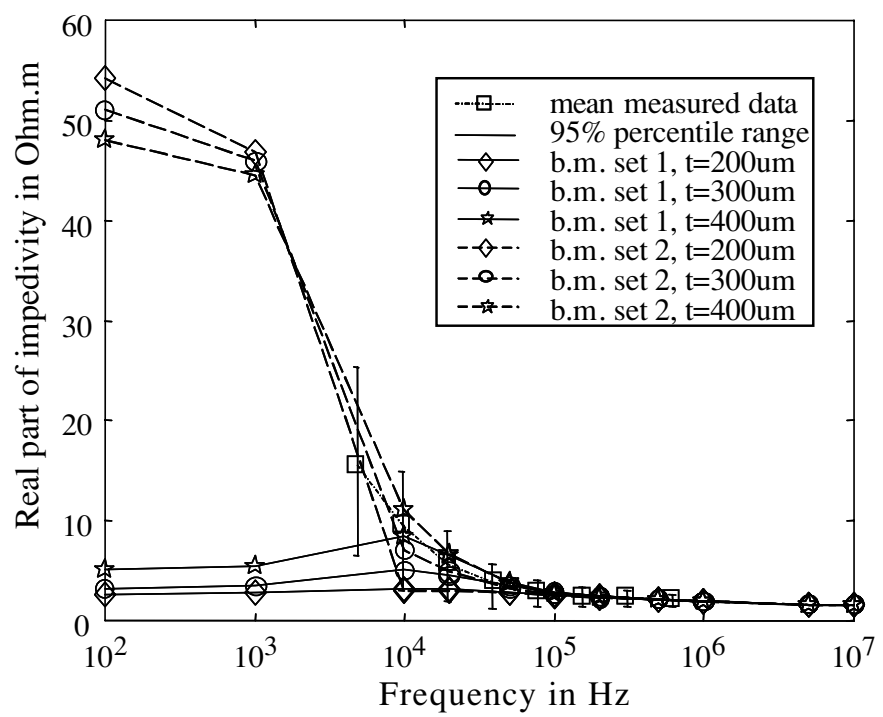

Figure 6. Effect of basement membrane (b $\mathrm{m}$ ) properties and epithelial thickness, $t$, on real component of modelled impedance spectrum.

at the points at which in vivo measurements were taken was carried out independently by a pathologist, without knowledge of the outcome of the impedance tests (Brown et al 2000).

Figure 7 shows the in vivo spectra, along with those obtained from a macroscopic tissue model containing an epithelium of variable thickness, with basement membrane properties derived from set (1) only. In this case, a conductive mucus layer of variable thickness $d m$, is included at the surface of the model, between the electrodes and the first epithelial layer. 


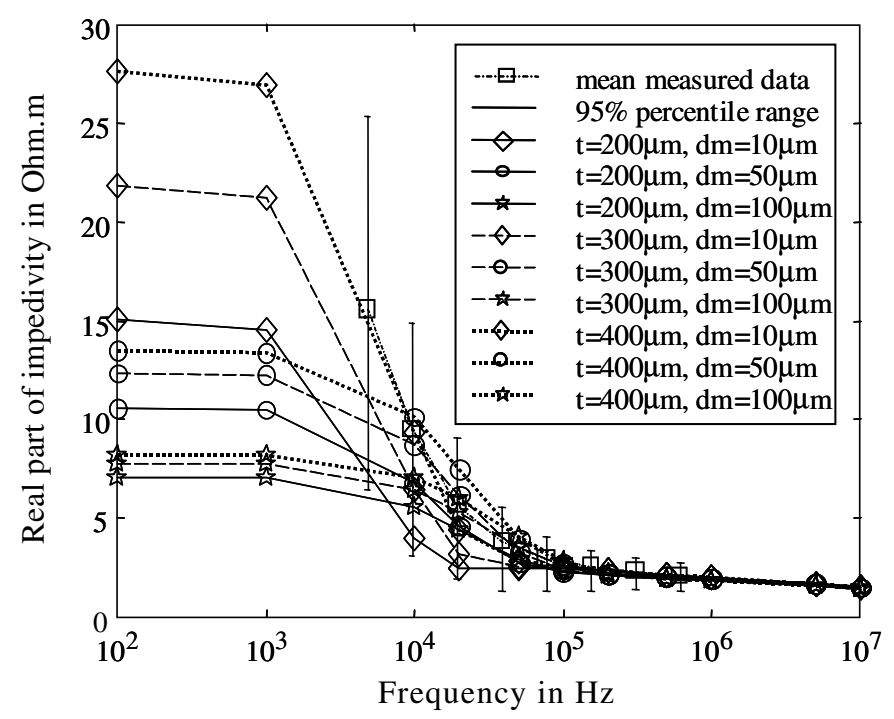

Figure 7. Effect of depth of surface mucus layer, $d m$, and epithelial thickness, $t$, on real component of modelled impedance spectrum using basement membrane properties set 2 .

Table 5. Percentage of total current flowing through each layer in and below epithelium of thickness $300 \mu \mathrm{m}$, using different basement membrane properties. $(\mathrm{S}=$ Superficial, $\mathrm{I}=$ intermediate, $\mathrm{P}=$ parabasal, B = Basal).

\begin{tabular}{|c|c|c|c|c|c|c|}
\hline \multirow[b]{2}{*}{ Layer } & \multicolumn{3}{|c|}{ Basement membrane properties set (1) } & \multicolumn{3}{|c|}{ Basement membrane properties set (2) } \\
\hline & $f=100 \mathrm{~Hz}$ & $f=10 \mathrm{kHz}$ & $f=1 \mathrm{MHz}$ & $f=100 \mathrm{~Hz}$ & $f=10 \mathrm{kHz}$ & $f=1 \mathrm{MHz}$ \\
\hline S & 1.2 & 4.6 & 7.1 & 3.7 & 9.4 & 7.2 \\
\hline I & 0.9 & 1.6 & 5.1 & 4.8 & 4.3 & 5.2 \\
\hline $\mathrm{P}$ & 1.5 & 1.7 & 6.3 & 14.1 & 5.1 & 6.4 \\
\hline $\mathrm{B}$ & 2.1 & 2.2 & 5.5 & 29.1 & 7.4 & 5.7 \\
\hline $\mathrm{BM}$ & 4.1 & 3.9 & 3.4 & 1.7 & 2.9 & 3.4 \\
\hline Stroma & 90.5 & 86.3 & 73.9 & 52.1 & 72.1 & 73.4 \\
\hline
\end{tabular}

\subsection{Analysis of current distribution}

Table 5 shows the calculated total current flowing in the models through each of the epithelial and underlying layers at frequencies of $100 \mathrm{~Hz}, 10 \mathrm{kHz}$ and $1 \mathrm{MHz}$ for the mean epithelial thickness of $300 \mu \mathrm{m}$ and basement membrane property sets (1) and (2). Table 6 shows current distributions for models including a surface mucus layer of variable thickness, for basement membrane property set (1) only at the same three frequencies.

\section{Discussion}

It is shown in figure 5 that the frequency-dependent characteristics of normal cervical squamous tissue measured with the surface tetrapolar electrode array, fall within the range of the predicted transfer impedivities of the four epithelial cell types across the frequency range considered. This suggests that if current flow was confined to the epithelium, the modelled macroscopic 
Table 6. Percentage of total current flowing through each layer in and below epithelium of thickness $300 \mu \mathrm{m}$, using basement membrane properties set (1) for model with variable thickness surface mucus layer. $(\mathrm{S}=$ Superficial, $\mathrm{I}=$ intermediate, $\mathrm{P}=$ parabasal, $\mathrm{B}=\mathrm{Basal}$ ).

\begin{tabular}{|c|c|c|c|c|c|c|c|c|c|}
\hline \multirow[b]{2}{*}{ Mucus depth } & \multicolumn{3}{|c|}{$f=100 \mathrm{~Hz}$} & \multicolumn{3}{|c|}{$f=10 \mathrm{kHz}$} & \multicolumn{3}{|c|}{$f=1 \mathrm{MHz}$} \\
\hline & $10 \mu \mathrm{m}$ & $50 \mu \mathrm{m}$ & $100 \mu \mathrm{m}$ & $10 \mu \mathrm{m}$ & $50 \mu \mathrm{m}$ & $100 \mu \mathrm{m}$ & $10 \mu \mathrm{m}$ & $50 \mu \mathrm{m}$ & $100 \mu \mathrm{m}$ \\
\hline Mucus & 32.5 & 60.2 & 68.9 & 17.8 & 49.5 & 61.3 & 2.6 & 10.8 & 19.5 \\
\hline S & 1.0 & 0.4 & 0.2 & 2.9 & 0.9 & 0.5 & 5.3 & 5.1 & 4.6 \\
\hline I & 0.7 & 0.3 & 0.2 & 1.2 & 0.4 & 0.2 & 5.6 & 5.3 & 4.7 \\
\hline $\mathrm{P}$ & 0.7 & 0.3 & 0.2 & 0.9 & 0.4 & 0.2 & 4.7 & 4.3 & 3.7 \\
\hline B & 1.2 & 0.5 & 0.3 & 1.6 & 0.7 & 0.4 & 5.3 & 4.8 & 4.1 \\
\hline BM & 2.3 & 1.0 & 0.6 & 2.9 & 1.4 & 0.9 & 3.4 & 3.0 & 2.6 \\
\hline Stroma & 59.0 & 31.8 & 23.2 & 71.4 & 42.2 & 30.8 & 74.4 & 67.1 & 60.2 \\
\hline
\end{tabular}

surface impedance spectra obtained using these properties should also agree closely with in vivo measurements. However, figure 6 shows that the modelled macroscopic spectra are extremely sensitive to the values of the material properties selected for the basement membrane layer. If we assume that the properties of this layer are similar to those of tendon across the whole frequency range, almost all the current penetrates beneath the epithelium and into the underlying stroma, as shown in table 5. In this case, the dispersion, (the reduction in the real part of the impedivity with frequency) predicted by our model is significantly smaller than that observed in the real data. The impedance curves generated by the model reflect the electrical characteristics of the stroma, which has a relatively high conductivity that changes little with frequency.

As expected, the fraction of current penetrating the basement membrane and flowing within the stroma is reduced when the basement membrane is assumed to be much less conductive. This effect is most pronounced at very low frequencies, when almost half of the total current flow occurs within the epithelium. Hence at these frequencies, the impedance characteristics are dominated by the relatively high impedance of the epithelial layers. As the current frequency is increased, the capacitive part of the conductance of the basement membrane increases, and more current is able to penetrate to the large, relatively low resistivity volume beneath. This results in a significant dispersion and the modelled spectrum falls within the $95 \%$ percentile range for the in vivo data, irrespective of the epithelial depth.

Our model was further refined by the addition of a thin, conductive surface layer, resembling mucus that is likely to be present on the surface of cervical epithelium. In this case, the properties used to model the basement membrane layer were those published in the literature for tendon, which are more likely to be a realistic representation of the collagenbased layer. From inspection of figure 7, it is immediately apparent that the fit between the modelled and measured impedance spectra is significantly improved by the inclusion of this layer. The mechanism for this improvement can be understood by inspection of the frequency-dependent current distribution for this model, summarized in table 6. At low frequencies, the current flow is divided between the surface mucus layer and the stroma, with negligible flow through the epithelial layers parallel to the surface. The division of current between the mucus and the stroma is dependent on the thickness of the surface layer. As the frequency is increased, a greater proportion of the current penetrates directly through the epithelium, and flows freely throughout the large conduction volume beneath, resulting in a significant reduction in impedance. The frequency at which this effect occurs is dependent on the thickness of the surface layer-the thinner the mucus, the lower the dispersion frequency. At this stage, current flow through the epithelium remains negligible. 
Finally, at higher frequencies, approximately $20 \%$ of the total current flow takes place through the epithelial layers in which we are interested. There is a corresponding reduction in current flowing through the surface layer, but most of the flow takes place through the deeper fibromuscular stroma. Inspection of figure 7 shows that there is considerable overlap in the impedance spectra obtained for models containing epithelia and mucus layers of different thicknesses. However, the best fit with the measured data is obtained for relatively thick epithelia (300-400 $\mu \mathrm{m})$ and thin surface layers $(10 \mu \mathrm{m})$.

We have shown that a reasonable fit between our modelled impedance spectra, and those obtained from in vivo measurements on normal cervical epithelium, can be achieved by the assumption of either of two scenarios: a basement membrane layer with a very high resistivity, that essentially acts as a capacitor, confining current to the epithelium at low frequencies, or a thin, conductive surface layer, which provides a low resistance path to the current at low frequencies, essentially short-circuiting the path through the tissue beneath. In the latter case, it is the epithelium itself that acts like a capacitor, with its resistance to current flow decreasing as the current frequency is increased.

Consideration of the biology of cervical tissue structure would probably suggest that it is the second of these scenarios that is more likely to be realistic. It seems quite reasonable to assume that the surface of epithelial tissue lying inside the cervical canal is coated with a very thin layer of mucus and hence the probe electrodes will not be in direct contact with the membranes of the superficial cells. However, there is little biological basis for assuming that the basement membrane, which consists of a network of collagen and other fibrous macromolecules, is likely to have the same electrical properties as lipid bi-layer structures associated with cellular membranes. Although there is no electrical data for this tissue published in the literature, and attempts to measure them in vitro have so far been unsuccessful, it is more likely that basement membrane will have properties similar to other biological materials containing collagen and other fibrous macromolecules.

In either case, we have shown that contrary to our initial assumptions, it is unlikely that the frequency-dependent properties of the epithelial layers, shown in figure 5, dominate the characteristic impedance spectra that we measure in vivo, but features associated with the macroscopic tissue structure are more important. However, previous work has shown that there is good separation between measurements made on normal and pathological tissue. The next logical step is therefore to investigate whether there is any biological evidence that these features are correlated with the progression of cervical pathology. For instance, it might be reasonable to assume that thicker mucus layers are associated with more advanced stages of CIN, where there is known to be a breakdown in tight junctions at the tissue surface, and an increase in the volume of extracellular fluid. This information can then be used to construct reliable models to aid diagnosis of cervical precancer.

\section{Acknowledgments}

The authors would like to thank the clinical staff of the Department of Obstetrics and Gynaecology and Sheffield Teaching Hospitals, and Professor M Wells of the Department of Histopathology for their help in carrying out the study on the electrical properties of unfixed cervical stroma.

\section{References}

Asami K T Y and Takashima S 1989 Dielectric properties of mouse lymphocytes and erythrocytes Biochim. Biophys. Acta 1010 49-55 
Bordi F, Cametti C, Rosi A and Calcabrini A 1993 Frequency-domain electrical-conductivity measurements of the passive electrical-properties of human-lymphocytes Biochim. Biophys. Acta 1153 77-88

Brown B H, Tidy J A, Boston K, Blackett A D, Smallwood R H and Sharp F 2000 Relation between tissue structure and imposed electrical current flow in cervical neoplasia Lancet 355 892-5

Cole K S 1968 Membranes, Ions and Impulses (Berkeley, CA: University of California Press)

Friedrich E R 1973 The normal morphology and ultrastructure of the cervix The Biology of the Cervix ed R J Blandau and K Moghissi (Chicago, IL: The University of Chicago Press)

Gabriel S, Lau R W and Gabriel C 1996a The dielectric properties of biological tissues: II. Measurements in the frequency range $10 \mathrm{~Hz}$ to $20 \mathrm{GHz}$ Phys. Med. Biol. 41 2251-69

Gabriel S, Lau R W and Gabriel C 1996b The dielectric properties of biological tissues: III. Parametric models for the dielectric spectrum of tissues Phys. Med. Biol. 41 2271-93

Geddes L A and Baker L E 1967 The specific resistance of biological material-a compedium of data for the biomedical engineer and physiologist Med. Biol. Eng. 5 271-93

Huang Y, Wang X B, Holzel R, Becker F F and Gascoyne P R C 1995 Electrorotational studies of the cytoplasmic dielectric-properties of friend murine erythroleukemia-cells Phys. Med. Biol. 40 1789-806

Irimajiri A, Doida Y, Hanai T and Inoyue A 1978 Passive electrical properties of cultured murine lymphoblast (L5178Y) with reference to its cytoplasmic membrane, nuclear envelope and intracellular phases J. Membr. Biol. 38209

Kotnik T and Miklavcic D 2000 Second-order model of membrane electric field induced by alternating external electric fields IEEE Trans. Biomed. Eng. 47 1074-81

Miller C E and Henriquez C S 1990 Finite element analysis of bioelectric phenomena Crit. Rev. Biomed. Eng. 18 207-33

Pethig R and Kell D B 1987 The passive electrical properties of biological systems: their significance in physiology, biophysics and biotechnology Phys. Med. Biol. 32 933-70

Schwan H P 1957 Electrical properties of tissue and cell suspensions Adv. Biol. Med. Phys. 5 147-209

Sorenson F B, Bichel P and Jakobsen A 1991 Stereological estimates of nuclear volume in squamous cell carcinoma of the uterine cervix and its precursors Virchows Archiv A Pathol. Anat. 418 225-33

Walker D C, Brown B H, Hose D R and Smallwood R H 2000 Modelling the electrical properties of normal and premalignant cervical tissue Electron. Lett. 36 1603-4

White F H and Gohari K 1984 Alterations in the volume of intercellular space between epithelial cells of the hamster cheek-pouch: quantitative studies of normal and carcinogen-treated tissues J. Oral Pathol. 13 224-54 